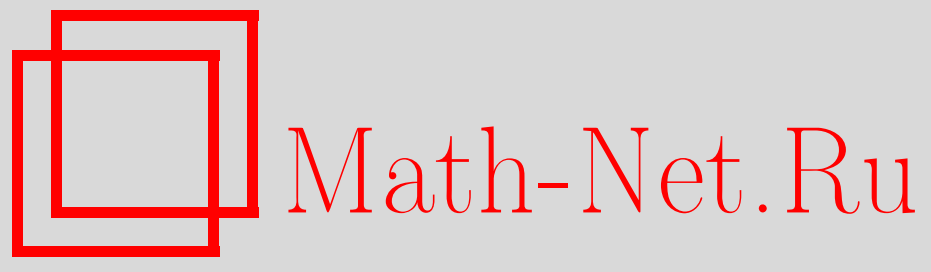

М. Я. Мазалов, О задаче Дирихле для полианалитических функций, Матем. сб., 2009, том 200, номер 10, 5980

DOI: https://doi.org/10.4213/sm7533

Использование Общероссийского математического портала Math-Net.Ru подразумевает, что вы прочитали и согласны с пользовательским соглашением http://www . mathnet.ru/rus/agreement

Параметры загрузки:

IP : 54.166 .219 .16

26 апреля 2023 г., 04:39:17

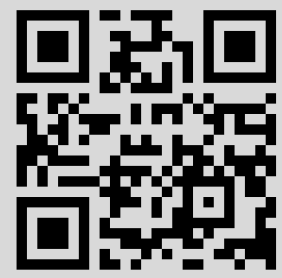




\section{М. Я. Мазалов}

\section{О задаче Дирихле для полианалитических функций}

Изучается связь между граничным поведением полианалитических функций и структурой границы. В частности, построена жорданова область с липшицевой границей, регулярная относительно задачи Дирихле в классе бианалитических функций.

Библиография: 14 названий.

Ключевые слова: полианалитические функции, граничные свойства, конструкция Лузина-Привалова, однолистные функции, лакунарные ряды, теорема Рудина-Карлесона.

\section{Введение. Формулировка результатов}

Напомним (см., например, [1]), что полианалитическими функииями порядка $n$ (кратко - $n$-аналитическими, при $n=2$ - бианалитическими) называются решения уравнения $\partial^{n} f / \partial \bar{z}^{n}=0$ на открытых подмножествах комплексной плоскости $\mathbb{C}$, где $\partial / \partial \bar{z}$ - оператор Коши-Римана. При этом каждая функция, $n$-аналитическая в некоторой области, однозначно представима в виде

$$
f(z)=\sum_{m=0}^{n-1} f_{m}(z) \bar{z}^{m},
$$

где все $f_{m}$ - функции, аналитические в той же области, называемые аналитическими компонентами $f$.

Пусть $G \subset \mathbb{C}$ - жорданова область со спрямляемой границей, Г - граничная дуга, $f$ - функция, $n$-аналитическая в области $G$ и имеющая почти всюду на дуге Г угловые граничные значения. Напомним, что граничная теорема единственности Лузина-Привалова [2; гл. 4, §2] и теорема Голубева-Привалова [2; гл. $3, \S 5]$ накладывают весьма жесткие условия на граничные значения аналитических функций. Рассмотрим ситуацию для $n$-аналитических функций при $n \geqslant 2$.

В случае аналитических дуг ситуация стандартна. Действительно, уравнение аналитической дуги Г имеет вид $\bar{z}=A(z)$, где $A(z)$ - функция, аналитическая в некоторой окрестности $\Gamma$, называемая функиией Швариа [3]. При этом (см., например, $[1 ; \S 4]$ ) угловые граничные значения функции $f$ на $\Gamma$ совпадают

Работа выполнена в рамках Программы поддержки ведущих научных школ РФ (грант № НШ-3877.2008.1). 
в соответствующих точках с угловыми граничными значениями аналитической согласованной функции

$$
f_{\Gamma}(z)=\sum_{m=0}^{n-1} f_{m}(z)(A(z))^{m} .
$$

Ситуация становится более сложной, если Г нигде не аналитична. Именно этот случай и будет основным в настоящей работе.

В $\S 1$ строится бесконечно гладкая кривая, на которой для полианалитических функций любого порядка граничная теорема единственности ЛузинаПривалова справедлива в такой же формулировке, как и для аналитических функций.

ПримеР 1. Существует жорданова область $D$, ограниченная бесконечно гладкой кривой $\partial D$ и обладающая следующим свойством. Пусть $D^{\prime} \subset D$ - произвольная жорданова область такая, что ее граничная дуга $\Gamma$ является частью $\partial D, f$ - функция, полианалитическая в $D^{\prime}$. Если $f$ имеет на подмножестве Г положительной длины угловые граничные значения, равные нулю, то $f \equiv 0$ в $D^{\prime}$.

Заметим, что $\partial D$ не может пересекаться ни с какой аналитической дугой на множестве положительной длины (в противном случае непостоянная бианалитическая функция $\bar{z}-A(z)$ равна нулю на этом множестве). Вместе с тем, существуют области с нигде не аналитической границей, в которых есть непостоянные бианалитические функции, равные нулю на границе [4].

В $\S 2-4$ исследуется вопрос о разрешимости краевой задачи Дирихле для полианалитических функций, поставленный, в частности, в [5; §4]. Пусть $H-$ функция, аналитическая в круге $K$, задаваемом неравенством $|w|<1$, и однолистно отображающая $K$ на жорданову область $G$ со спрямляемой границей $\partial G$, причем $\left|H^{\prime}(w)\right|>c_{0}>0$ в $K$. Пусть $C(\partial G)$ - банахово пространство функций, непрерывных на $\partial G$, с равномерной нормой; $S_{n}=S_{n}(\partial G, G)-$ подпространство $C(\partial G)$, элементами которого являются граничные значения функций, $n$-аналитических в $G$ и непрерывных в замкнутой области $\bar{G} ; S=$ $\bigcup_{n} S_{n}$. В $\S 2$ будет доказана следующая теорема.

ТЕОРема 1. Если выполнено условие

$$
\iint_{K}\left|H^{\prime \prime}(w)\right| r d r d \varphi<\infty
$$

(где $\left.w=r e^{i \varphi}\right)$, то $S$ имеет первую категорию в $C(\partial G)$.

Так как $C(\partial G)$ - полное метрическое пространство, то по теореме Бэра о категории (см., например, [6; гл. 2, п. 2.2]) имеем $S \neq C(\partial G)$. Заметим, что условие $(0.2)$, в частности, выполняется, если $\partial G$ - Дини-гладкая кривая (лемма 2.2); этот факт по существу вытекает из теоремы Варшавского (см. [7; теорема 10.2]). Имеет место и локальное утверждение, уточняющее теорему 1, это теорема 2 . 
Теорему 1 интересно сопоставить с результатом Федоровского (см. [8; теорема 1], $[5 ; \S 2$, п. 2.6]): для “большинства" областей $G$, в том числе и с аналитической границей (кроме весьма специальных областей, ограниченных неванлинновскими контурами) $S_{2}(\partial G, G)$ является всюду плотным подпространством $C(\partial G)$.

Возникает естественный вопрос: можно ли в теореме 1 отбросить условие (0.2)? Пример, построенный в $\S 3-4$ и являющийся наиболее существенным результатом настоящей работы, показывает, что этого сделать нельзя.

ПримеР 2. Существует жорданова область $G$ с липшицевой границей такая, что $S_{2}(\partial G, G)=C(\partial G)$.

В $\S 3$ с помощью лакунарных рядов строятся вспомогательные функции и основным результатом здесь является теорема 3. Построение примера завершается в $\S 4$, где приводится геометрическая конструкция искомой области. В доказательстве существенно используются теорема Рудина-Карлесона об интерполяционных множествах пика для непрерывных аналитических функций (см. [9], [10]) и методы функционального анализа.

Автор выражает благодарность М.С. Мельникову, П.В. Парамонову и К. Ю. Федоровскому за постановку задачи и полезные обсуждения.

\section{§ 1. Построение примера 1}

Построим функцию $H$, аналитическую в круге $K$ и однолистно отображающую $K$ на требуемую область $D$.

Далее $B=B(a, r)$ - это открытый круг с центром в точке $a$ и радиусом $r ; \lambda B$ - круг с тем же центром и радиусом $\lambda r$. Пусть $\left\{B_{j}\right\}=\left\{B\left(a_{j}, r_{j}\right)\right\}$, $j=1,2, \ldots,-$ счетное семейство кругов таких, что $2 B_{j}$ попарно внешние, расположены вне $K$ и $r_{j}<1 / 2$ для всех $j$. При этом выполнены следующие два условия:

(1) условие Бляшке, т.е. $\sum_{j}\left(\left|a_{j}\right|-1\right)<\infty$;

(2) замыкание множества $\left\{a_{j}\right\}$ содержит единичную окружность $\partial K$.

Рассмотрим два стандартных следствия условия (1).

СлЕДСтвиЕ 1. Зафиксируем произвольное $\psi, 0<\psi<\pi / 2$, и для любой точки $e^{i \varphi}$ возъмем угол $V_{\psi}\left(e^{i \varphi}\right)$, образованный лучами $z=e^{i \varphi}+t e^{i(\varphi \pm \psi)}, t \geqslant 0$. Тогда для почти всех точек $\partial K$ соответствующий угол $V_{\psi}\left(e^{i \varphi}\right)$ пересекает не более чем конечное множество кругов $B_{j}$.

Действительно, для каждого достаточно большого номера $j$ возьмем криволинейный равнобедренный треугольник, образованный углом при вершине величины $2 \psi$, описанным вокруг $B_{j}$, и дугой, отсекаемой этим углом на $\partial K$, в качестве основания. Тогда в силу (1) сумма характеристических функций оснований таких треугольников почти всюду конечна на $\partial K$.

Пусть $K^{\prime}=(|w|>1) \backslash \bigcup_{j} B_{j}$. Следствие 1 позволяет рассматривать угловые граничные значения почти всюду на $\partial K$ функций, заданных на $K^{\prime}$. 


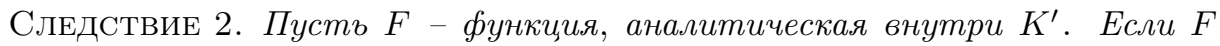
имеет на подмножестве дК положительной длины угловые граничные значения, равные нулю, то $F \equiv 0$.

Доказательство следствия 2 получается повторением рассуждений из построения Лузина-Привалова [2; гл. 4, § 2, п. 2.4] или [11; гл. 3, § D] (так же, как и для мероморфных функций с полюсами в $B_{j}$ ).

Определим функцию $H$ следующим образом:

$$
H(w)=w+\sum_{j, k} \frac{\varepsilon_{j, k}}{w-w_{j, k}},
$$

где для фиксированного $j$ множество $\left\{w_{j, k}\right\}$ - счетное множество изолированных точек, расположенных в $(1 / 2) B_{j}, \sum_{k}\left|\varepsilon_{j, k}\right|<\left(r_{j}\right)^{j+2}, \varepsilon_{j, k} \neq 0$ при всех $j$ и $k$.

ЛЕмма 1.1. Функиия $Н$ обладает следующими свойствами:

1) $Н$ является аналитической всюду вне замыкания множества $\left\{w_{j, k}\right\}$ (в частности, аналитической в $K$ );

2) сужение $H$ на $K^{\prime}$ является непрерывной функцией, причем производные $H$ любого порядка непрерывно продолжаются изнутри $K$ на $\partial K$;

3) на множестве $K^{\prime}$ верна оченка $|H(w)-H(1 / \bar{w})| \leqslant 6(1-|w|)$.

4) $H$ однолистно отображает $K$ на жорданову область;

5) $Н$ имеет на $\partial K$ вне $K$ почти всюду угловые граничные значения.

ДокАзАтельство. Аналитичность $H$ вне замыкания $\left\{w_{j, k}\right\}$ следует из сходимости ряда $\sum_{j, k}\left|\varepsilon_{j, k}\right|$, а свойство 2) - из следующих оценок на $K^{\prime}$ :

$$
\sum_{j, k}\left|\left(\frac{\varepsilon_{j, k}}{w-w_{j, k}}\right)^{(m)}\right|<m ! 2^{m+1} \sum_{j=1}^{\infty}\left(r_{j}\right)^{j+1-m}<\infty .
$$

Аналогично, на множестве $K^{\prime}$ справедлива оценка

$$
\left|H(w)-H\left(\frac{1}{\bar{w}}\right)\right| \leqslant\left|w-\frac{1}{\bar{w}}\right|\left(1+2 \sum_{j=1}^{\infty}\left(r_{j}\right)^{j}\right) \leqslant 6(1-|w|) .
$$

При $|w|<1$ имеем место оценка

$$
\left|H^{\prime}(w)-1\right|<\frac{4}{9} \sum_{j=1}^{\infty}\left(r_{j}\right)^{j}<\frac{4}{9}
$$

откуда следует 4). Свойство 5) вытекает из 2) и следствия 1 условия 1). Лемма доказана.

Обозначим через $D$ жорданову область с бесконечно гладкой границей, на которую в силу леммы 1.1 функция $H$ отображает $K$, и покажем, что эта область - искомая. Не нарушая общности рассуждений, будем считать, что область $D^{\prime}$ - образ пересечения окрестности произвольной точки $e^{i \varphi}$ с $K$, а дуга $Г$ соответственно - образ пересечения этой окрестности с $\partial K$. Пусть $D_{1}-$ прообраз $D^{\prime}$, а $\Gamma_{1}-$ прообраз $\Gamma$. 
ЛЕмма 1.2. Пусть $f$ - функция, -аналитическая в $D^{\prime}$. Если $f$ принимает на подмножестве Г положительной длины угловые граничные значения, равные нулю, то $f \equiv 0$ в $D^{\prime}$.

ДоказАтельство. Пусть $w_{0} \in \Gamma_{1}, z_{0}=H\left(w_{0}\right), f(z)-$ функция, $n$-аналитическая в $D^{\prime}$ и имеющая в точке $z_{0}$ угловое граничное значение, которое обозначим через $f\left(z_{0}\right)$. Тогда из аналогов неравенств Коши следует, что каждая из функций $\left(z-z_{0}\right)^{m}\left(\partial^{m} f / \partial \bar{z}^{m}\right), m=1,2, \ldots$, имеет в точке $z_{0}$ угловое граничное значение, равное нулю. (Действительно $[1 ; \S 1$, п. 1.4, формула 16], если функция $g n$-аналитична в круге $B(a, \delta)$ и ее модуль ограничен в этом круге постоянной $M$, то в центре круга $\zeta$ имеют место неравенства

$$
\left|\nabla^{m} g(\zeta)\right| \leqslant A(n, m) \frac{M}{\delta^{m}},
$$

и остается применить (1.2) к функции $g(z)=f(z)-f\left(z_{0}\right)$.)

Запишем $f(z)$ в виде $(0.1)$ и рассмотрим ее прообраз в $D_{1}\left(w=H^{-1}(z)\right)$ :

$$
F(w)=\sum_{m=0}^{n-1} f_{m}(H(w)) \overline{H(w)}^{m} .
$$

Напомним, что из неравенства $\left|H^{\prime}(w)\right|>c_{0}>0$ (которое здесь заведомо выполняется при $\left.c_{0}=5 / 9\right)$ в силу теоремы Кёбе об искажении (см., например, [12; гл. $2, \S 4$, теорема 2]) следует, что

$$
\sup _{w \in K} \frac{\operatorname{dist}(w, \partial K)}{\operatorname{dist}(H(w), \partial D)}<\infty .
$$

Отсюда заключаем, что для каждой формальной производной $\partial^{m} F / \partial \bar{H}^{m}$ (прообраза $\partial^{m} f / \partial \bar{z}^{m}$ ) при $m=1,2, \ldots$ имеет место равенство

$$
\lim _{w \rightarrow w_{0}}\left(\left(w-w_{0}\right)^{m} \frac{\partial^{m} F}{\partial \bar{H}^{m}}(w)\right)=0,
$$

если $w$ стремится к $w_{0}=H^{-1}\left(z_{0}\right)$ по некасательному направлению.

Для $H(w)$ из (1.1) рассмотрим функцию $H_{*}(w)=\overline{H(1 / \bar{w})}$ :

$$
H_{*}(w)=\frac{1}{w}+\sum_{j, k} \frac{\overline{\varepsilon_{j, k}}}{w^{-1}-\overline{w_{j, k}}} .
$$

Свойства функции $H_{*}(w)$ с точностью до инверсии аналогичны свойствам $H(w)$, указанным в лемме 1.1. В частности, $H_{*}(w)$ аналитична всюду вне $w=0$ и замыкания множества $\left\{1 / \overline{w_{j, k}}\right\}$, непрерывна на множестве $K^{\prime \prime}=(1 / 2 \leqslant$ $|w| \leqslant 1) \backslash \bigcup_{j} B_{j}^{\prime}$ (где $B_{j}^{\prime}-$ круги, полученные из $B_{j}$ с помощью инверсии) и имеет почти всюду на $\partial K$ изнутри $K$ угловые граничные значения. Пусть $w \in K$, причем $w \neq 0, w \neq 1 / \overline{w_{j, k}}$, а $\Phi-$ следующая функция:

$$
\Phi(w)=\sum_{m=0}^{n-1} f_{m}(H(w))\left(H_{*}(w)\right)^{m} .
$$


Применив формулу Тейлора для многочленов, из (1.3) получим

$$
\Phi(w)=F(w)+\sum_{m=1}^{n-1} \frac{1}{m !}\left(H_{*}(w)-\overline{H(w)}\right)^{m} \frac{\partial^{m} F}{\partial \bar{H}^{m}}(w) .
$$

Разность $H_{*}(w)-\overline{H(w)}$ оценивается на $K^{\prime \prime}$ так же, как и в утверждении 3$)$ леммы 1.1 на $K^{\prime}$ оценивается разность $H(w)-H(1 / \bar{w})$. Отсюда в силу $(1.5)$ получим, что угловые граничные значения функций $F(w)$ и $\Phi(w)$ на $\partial K$ (внутри $K)$ в случае существования почти всюду совпадают.

Завершим доказательство леммы 1.2. Пусть функция $f(z)$ удовлетворяет условиям этой леммы. Тогда функция $F(w)$ и, следовательно, $\Phi(w)$ имеет на подмножестве $\Gamma_{1}$ положительной длины угловые граничные значения, равные нулю. Применив инверсию и воспользовавшись следствием 2 условия (1) (условия Бляшке), получим, что $\Phi \equiv 0$.

Чтобы показать, что $f \equiv 0$, достаточно убедиться в том, что $f_{n-1} \equiv 0$, где $f_{n-1}$ - старшая аналитическая компонента $f$. Пусть $\gamma$ - контур, охватывающий только одну из точек $1 / \overline{w_{j, k}}$ (все остальные точки расположены вне контура). Тогда в силу (1.6) и (1.7) имеем

$$
\begin{aligned}
0 & =\oint_{\gamma} \frac{\Phi(w)\left(w^{-1}-\overline{w_{j, k}}\right)^{n-2}}{\overline{\varepsilon, k}^{n-1}} d w=\oint_{\gamma} \frac{f_{n-1}(H(w))}{w^{-1}-\overline{w_{j, k}}} d w \\
& =-\frac{2 \pi i}{{\overline{w_{j, k}}}^{2}} f_{n-1}\left(H\left(\frac{1}{\overline{w_{j, k}}}\right)\right) .
\end{aligned}
$$

Так как в силу определения $H$ и условия (2), наложенного на $\left\{a_{j}\right\}$, множество $\left\{1 / \overline{w_{j, k}}\right\}$ имеет предельные точки внутри $D_{1}$, то по внутренней теореме единственности аналитических функций получаем, что $f_{n-1} \equiv 0$. Лемма доказана.

\section{§ 2. Доказательство теоремы 1}

Далее через $A, A_{1}, A_{2}, \ldots$ будем обозначать положительные постоянные, каждая из которых в разных соотношениях может принимать различные значения.

Пусть $G$ - жорданова область со спрямляемой границей, $f(z)$ - функция, $n$-аналитическая в $G$ и непрерывная в замкнутой области $\bar{G}$ (т.е. $f \in A_{n}(\bar{G})$ ), $F(w)$ - соответствующая $f(z)$ функция из (1.3) (т.е. $\left.F \in \widetilde{A_{n}}(\bar{K})\right), H$ - функция, аналитическая в круге $K$ и однолистно отображающая $K$ на $G$, причем выполнено условие $(0.2)$ и $\left|H^{\prime}(w)\right|>c_{0}>0$ в $K$.

Пусть $M=\max _{|w| \leqslant 1} F(w)$. Теорема 1 немедленно вытекает из следующей оценки, доказываемой в лемме 2.1:

$$
\frac{1}{2 \pi}\left|\int_{|w|=1} F(w) w^{N} d w\right| \leqslant \alpha_{N},
$$

где $N \in \mathbb{N}, \alpha_{N}=\alpha_{N}(M, n, H)$ и $\lim _{N \rightarrow \infty} \alpha_{N}=0$ (существенно, что $\alpha_{N}$ не зависит от функции $F$ ). 
Действительно, рассмотрим множество $S_{n, p}$ функций $F\left(e^{i \varphi}\right)$, являющихся граничными значениями функций $F \in \widetilde{A_{n}}(\bar{K})$ таких, что $|F(w)| \leqslant p$ в $K$, где $n$ и $p \in \mathbb{N}$ фиксированы. Пусть $U$ - произвольный шар пространства $C(\partial K)$ с центром $g$ радиуса $\varepsilon, P$ - тригонометрический многочлен вида $\sum_{|k| \leqslant n_{0}} c_{k} e^{i k \varphi}$ такой, что $\|g-P\|_{\mathrm{L}_{\infty}}<\varepsilon / 3$. В силу (2.1) можно взять $N$ таким, что $N>n_{0}$ и $\alpha_{N}=\alpha_{N}(p, n, H)<\varepsilon / 6$. Тогда, как нетрудно проверить, шар с центром в точке $P+e^{-i(N+1) \varphi} \varepsilon / 3$ радиуса $\varepsilon / 12$ содержится в $U$ и не содержит функций из $S_{n, p}$. Следовательно, множества $S_{n, p}$ нигде не плотны в пространстве $C(\partial K)$.

Лемма 2.1. В формуле (2.1) при достаточно больших $N$ можно взять

$$
\alpha_{N}=A(M, n, H) \iint_{K}\left|H^{\prime \prime}(w)\right| r^{N-n+1} r d r d \varphi .
$$

ДокАЗАтельство. Напомним, что функция $F(w)$ бесконечно дифференцируема в круге $|w| \leqslant R, R<1$. Интегрируя по частям, получим следующее тождество $(k \in \mathbb{N})$ :

$$
\iint_{|w| \leqslant R} k\left(R^{2}-r^{2}\right)^{k-1} F(w) w^{N} r d r d \varphi=\iint_{|w| \leqslant R}\left(R^{2}-r^{2}\right)^{k} \frac{\partial F}{\partial \bar{w}} w^{N-1} r d r d \varphi .
$$

Из формулы Грина и (2.3) следует, что при $N \geqslant n-1$ и $1 \leqslant m \leqslant n$

$$
\begin{aligned}
\int_{|w|=R} F(w) w^{N} d w & =2 i \iint_{|w| \leqslant R} \frac{\partial F}{\partial \bar{w}} w^{N} r d r d \varphi \\
& =\frac{2 i}{(m-1) !} \iint_{|w| \leqslant R}\left(R^{2}-r^{2}\right)^{m-1} \frac{\partial^{m} F}{\partial \bar{w}^{m}} w^{N-m+1} r d r d \varphi
\end{aligned}
$$

Сначала рассмотрим случай функций $F \in \widetilde{A_{2}}(\bar{K})$. Здесь в силу условия

$$
\frac{\partial^{2} F}{\partial \bar{H}^{2}} \equiv 0
$$

получим

$$
\begin{aligned}
\int_{|w|=R} F(w) w^{N} d w & =2 i \iint_{|w| \leqslant R}\left(R^{2}-r^{2}\right) \frac{\partial^{2} F}{\partial \bar{w}^{2}} w^{N-1} r d r d \varphi \\
& =2 i \iint_{|w| \leqslant R}\left(R^{2}-r^{2}\right) \frac{\partial F}{\partial \bar{H}}(w) \overline{H^{\prime \prime}(w)} w^{N-1} r d r d \varphi .
\end{aligned}
$$

Напомним, что в силу (1.2) имеем

$$
\left|\frac{\partial f}{\partial \bar{z}}(z)\right| \leqslant A(M) \operatorname{dist}(z, \partial G)^{-1} .
$$

Отсюда, используя оценки $\left|H^{\prime}(w)\right|>c_{0}>0$ и (1.4), получим

$$
\left|\frac{\partial F}{\partial \bar{H}}\left(r e^{i \varphi}\right)\right| \leqslant A(M, H)(1-r)^{-1} .
$$

Переходя к пределу при $R \rightarrow 1$, получим (2.2). 
При $n>2$ рассуждаем аналогично. Именно, последовательно вычисляя

$$
\begin{gathered}
\frac{\partial^{2} F}{\partial \bar{w}^{2}}=\frac{\partial F}{\partial \bar{H}} \overline{H^{\prime \prime}(w)}+\frac{\partial^{2} F}{\partial \bar{H}^{2}}{\overline{H^{\prime}(w)}}^{2} \\
\frac{\partial}{\partial \bar{w}}\left[\frac{\partial^{2} F}{\partial \bar{H}^{2}}{\overline{H^{\prime}(w)}}^{2}\right], \quad \ldots, \quad \frac{\partial}{\partial \bar{w}}\left[\frac{\partial^{n-1} F}{\partial \bar{H}^{n-1}} \bar{H}^{\prime}(w)^{n-1}\right]
\end{gathered}
$$

и учитывая неравенства

$$
\left|\frac{\partial^{m} F}{\partial \overline{H^{m}}}\left(r e^{i \varphi}\right)\right| \leqslant A(M, n, H)(1-r)^{-m}
$$

при $m<n$, а также то, что

$$
\frac{\partial^{n} F}{\partial \bar{H}^{n}} \equiv 0
$$

получим (2.2).

Лемма доказана, и тем самым доказана теорема 1.

Теорема 2. Пусть выполнены условия теоремы 1; ГС $С G$ - замкнутая дуга. Тогда множество функиий, являющихся граничными значениями на Г функциц из $A_{n}(\bar{G})$, имеет первую категорию в пространстве $C(\Gamma)$.

Ограничимся указанием тех несложных изменений, которые нужно внести в доказательство теоремы 1, чтобы получить доказательство теоремы 2 .

Действительно, нужно (в отличие от теоремы 1) рассмотреть функцию $F(w)$ не в круге, а в подходящем секторе $\Sigma$, ограниченном прямыми $\bar{w}=\zeta_{1} w$, $\bar{w}=\zeta_{2} w\left(\left|\zeta_{1}\right|=\left|\zeta_{2}\right|=1\right)$ и дугой окружности $|w|=R$; применить $(2.4)$ к вспомогательной функции $\Psi(w)=\left(\bar{w}-\zeta_{1} w\right)^{n}\left(\bar{w}-\zeta_{2} w\right)^{n} F(w)$, равной нулю на указанных прямых:

$$
\begin{aligned}
\int_{\partial \Sigma} \Psi(w) w^{N} d w & =2 i \iint_{\Sigma} \frac{\partial \Psi}{\partial \bar{w}} w^{N} r d r d \varphi \\
& =\frac{2 i}{(m-1) !} \iint_{\Sigma}\left(R^{2}-r^{2}\right)^{m-1} \frac{\partial^{m} \Psi}{\partial \bar{w}^{m}} w^{N-m+1} r d r d \varphi
\end{aligned}
$$

что приводит к оценке, аналогичной (2.1) для соответствующей дуги $\partial K$, и, следовательно, позволяет доказать теорему 2.

Покажем, что условия теоремы 1 выполняются для любой жордановой области, ограниченной Дини-гладкой кривой. Напомним [7; §10.1], что кривая называется Дини-гладкой, если ее угол наклона касательной $\beta(s)$ как функция длины дуги $s$ удовлетворяет условию $\left|\beta\left(s_{2}\right)-\beta\left(s_{1}\right)\right|<\widetilde{\omega}\left(\left|s_{2}-s_{1}\right|\right)$, где для модуля непрерывности $\widetilde{\omega}(\delta)$ выполнено условие Дини

$$
\int_{0}^{1} \frac{\widetilde{\omega}(\delta)}{\delta} d \delta<\infty
$$

Напомним, что имеет место теорема Варшавского (см. [7; теорема 10.2]): если $G$ - жорданова область с Дини-гладкой границей, то функция $H^{\prime}$ непреръвна в $\bar{K}$, причем $\left|H^{\prime}(w)\right|>c_{0}>0$ в $\bar{K}$. 
Указанная теорема по существу содержит следующее утверждение.

Лемма 2.2. Если $G$ - жорданова область с Дини-гладкой границей $\partial G$, то выполнено условие (0.2).

ДокАЗАтельство. Пусть $\nu(t)=\arg H^{\prime}\left(e^{i t}\right)$. Напомним, что по теореме Линделёфа (см. [7; теорема 10.1]) в силу гладкости $\partial G$ функция $\arg H^{\prime}(w)$ непрерывна в $\bar{K}$. По теореме Смирнова (см. [7; лемма 10.1]) и формуле (11) (см. [7; §10.1]) модуль непрерывности $\omega(\delta)$ функции $\nu(t)$ допускает оценку $\omega(\delta) \leqslant \delta+\widetilde{\omega}(A \sqrt{\delta})$ (где $A=A(H)$; напомним, что $\widetilde{\omega}(\delta)$ - модуль непрерывности функции $\beta(s)$, удовлетворяющий условию Дини). Отсюда легко вывести, что $\omega(\delta)$ также удовлетворяет условию Дини.

Пусть $w=r e^{i \varphi} \in K, \delta=1-r$. Из формулы Шварца следует, что

$$
\log H^{\prime}(w)=\log H^{\prime}(0)+\frac{i}{2 \pi} \int_{0}^{2 \pi} \frac{e^{i t}+w}{e^{i t}-w} \nu(t) d t .
$$

Дифференцируя по $w$ под знаком интеграла, имеем

$$
\left|H^{\prime \prime}(w)\right| \leqslant \frac{1}{2 \pi} \max _{\bar{K}}\left|H^{\prime}(w)\right|\left|\int_{0}^{2 \pi} \frac{2 e^{i t}[\nu(t)-\nu(\varphi)]}{\left(e^{i t}-w\right)^{2}} d t\right| .
$$

Разбивая интеграл в правой части на сумму интегралов по дугам $\partial K$, длины которых соизмеримы с $2^{j} \delta, j=1,2, \ldots$, получаем

$$
\left|H^{\prime \prime}(w)\right| \leqslant A\left(\omega(\pi)+\delta^{-1} \sum_{j=0}^{[\log (1 / \delta)]} \frac{\omega\left(2^{j} \delta\right)}{2^{j}}\right),
$$

где $A=A(H)$.

Отсюда, интегрируя $\left|H^{\prime \prime}(w)\right|$ по кольцам $\left\{1-2^{j+1} \delta \leqslant|w| \leqslant 1-2^{j} \delta\right\}, j=$ $0,1,2, \ldots$, находим, что

$$
\iint_{|w|<1-\delta}\left|H^{\prime \prime}(w)\right| r d r d \varphi \leqslant A \pi \omega(\pi)+A_{1} \sum_{k=0}^{[\log (1 / \delta)]} \sum_{j=k}^{[\log (1 / \delta)]} \frac{\omega\left(2^{j} \delta\right)}{2^{j-k}} .
$$

Двойная сумма в правой части неравенства не превосходит

$$
2 A_{1} \sum_{k=0}^{[\log (1 / \delta)]} \omega\left(2^{k} \delta\right)
$$

и остается равномерно ограниченной при $\delta \rightarrow 0$, так как

$$
\int_{0}^{1} \frac{\omega(x)}{x} d x<\infty
$$

Лемма доказана. 


\section{§ 3. Пример 2: построение вспомогательных функций}

Будем использовать лакунарные ряды вида

$$
h(w)=\sum_{k=1}^{\infty} \frac{\beta_{k}}{\alpha_{k}} w^{\alpha_{k}},
$$

где $\alpha_{k} \in \mathbb{N}, \alpha_{k+1} / \alpha_{k} \geqslant 2, \alpha_{k+1} / \alpha_{k} \nearrow \infty$ и $\beta_{k} \searrow 0$ при $k \rightarrow \infty$. Ясно, что в этом случае функция $h$ непрерывна в $\bar{K}$. Сделаем ряд замечаний, поясняющих выбор $\beta_{k}$.

1) Нетрудно убедиться, что

$$
\iint_{K}\left|h^{\prime \prime}(w)\right| r d r d \varphi \leqslant A \sum_{k=1}^{\infty}\left|\beta_{k}\right|
$$

и ограниченность $h^{\prime}(w)$ в $K$ равносильна сходимости ряда $\sum_{k=1}^{\infty}\left|\beta_{k}\right|$.

2) Функция $h^{\prime}(w)$ тогда и только тогда имеет почти всюду на $\partial K$ угловые граничные значения, когда $\sum_{k=1}^{\infty}\left|\beta_{k}\right|^{2}<\infty$ (см., например, [2; §10]).

3) Условие $\sum_{k=1}^{\infty}\left|\beta_{k}\right|=\infty$ несовместимо с однолистностью $h$ (см. [7; § 5.4]).

Поэтому будем считать, что в (3.1) дополнительно выполнены условия

$$
\sum_{k=1}^{\infty}\left|\beta_{k}\right|=\infty, \quad \sum_{k=1}^{\infty}\left|\beta_{k}\right|^{2}<\infty
$$

и возьмем $\beta_{k}=1 / k$. Сначала проведем построение в $K$, не требуя однолистности (в качестве результата получим теорему 3 ). В $\S 4$ проведем дополнительное построение на подходящем подмножестве $K$.

Рассмотрим следующую функцию:

$$
h_{k_{0}}(w)=w+\sum_{k=k_{0}}^{\infty} \frac{1}{k \alpha_{k}} w^{\alpha_{k}},
$$

где $k_{0} \in \mathbb{N}$ (за счет выбора $k_{0} \mathrm{~L}_{2}$-норму функции $h_{k_{0}}^{\prime}(w)-1$ на $\partial K$ можно сделать как угодно малой). Последовательность $\left\{\alpha_{k}\right\}$ будем считать настолько разреженной, что

$$
\sum_{n=2}^{\infty} \sum_{k=1}^{n-1} \frac{\alpha_{k}}{\alpha_{n}}<\infty
$$

(достаточно взять $\alpha_{k}=2^{k^{2}}$ ).

Заметим, что за счет разреженности $\alpha_{k}$ интеграл

$$
\iint_{|w| \leqslant r}\left|h^{\prime \prime}(w)\right| r d r d \varphi
$$

может расходиться как угодно медленно при $r / 1$. 
Лемма 3.1. Пусть $h(w)=h_{k_{0}}(w)$ из (3.2) или $h(w)=h_{k_{0}}(w)-w$. Тогда для любого $N_{0} \in \mathbb{Z}$ и для любого $\varepsilon>0$ существуют многочлень $\Phi_{1}(w)$ и $\Phi_{2}(w)$ такие, что имеют место следующие оченки:

1) $\left|F(w)-w^{-N_{0}}\right|<\varepsilon n p u|w|=1$, где $F(w)=\Phi_{1}(w) \overline{h(w)}-\Phi_{2}(w)$;

2) $|F(w)|<1+\varepsilon n p u|w| \leqslant 1$;

3) $\max \left(\left|\Phi_{1}(w)\right|,\left|\Phi_{2}(w)\right|\right)<\varepsilon(1-|w|)^{-1} n p u|w|<1$;

4) $|F(w)|<\varepsilon n p u|w|<1-\varepsilon$.

ДокАЗАтЕЛьство. Заметим, что достаточно установить оценки 1)-3) при $N_{0} \in \mathbb{N}$. Оценка 4) и случай остальных $N_{0}$ легко получаются отсюда умножением $F(w)$ на $w^{k}$ при подходящем $k \in \mathbb{N}$. Рассмотрим случай $h(w)=h_{k_{0}}(w)$ (случай $h_{k_{0}}(w)-w$ требует лишь тривиальных изменений).

Пусть $\left\{\varepsilon_{k}\right\}, \varepsilon_{k} \searrow 0,-$ последовательность, удовлетворяющая следующим двум условиям:

$$
\text { 1) } \lim _{k \rightarrow \infty} \varepsilon_{k} \log k=0 ; \quad \text { 2) } \sum_{k=1}^{\infty} \frac{\varepsilon_{k}}{k}=\infty
$$

(при $k>2$ достаточно взять $\left.\varepsilon_{k}=(\log k \log \log k)^{-1}\right)$.

Возьмем для $\varepsilon_{k}$ из (3.4) многочлен

$$
\Phi_{1}(w)=\sum_{k=k_{1}}^{k_{2}} \varepsilon_{k} \alpha_{k} w^{\alpha_{k}-N_{0}},
$$

где $k_{1}>k_{0}$ столь велико, что $\alpha_{k+1}-\alpha_{k}-N_{0}>(1 / 2) \alpha_{k+1}$ при $k \geqslant k_{1}$, и для подходящего $k_{2}$ имеем $\left|1-\sum_{k=k_{1}}^{k_{2}}\left(\varepsilon_{k} / k\right)\right|<\varepsilon / 2$. Рассмотрим функцию

$$
\Phi_{1}(w) \overline{h(w)}=\sum_{k=k_{1}}^{k_{2}} \varepsilon_{k} \alpha_{k} w^{\alpha_{k}-N_{0}}\left(\bar{w}+\sum_{m=k_{0}}^{\infty} \frac{1}{m \alpha_{m}} \bar{w}^{\alpha_{m}}\right) .
$$

Разобьем произведение на сумму следующих трех слагаемых.

1) $m=k$ :

$$
F_{1}(w)=\sum_{k=k_{1}}^{k_{2}} \frac{\varepsilon_{k}}{k} w^{\alpha_{k}-N_{0}} \bar{w}^{\alpha_{k}} .
$$

Ясно, что $\left|F_{1}(w)-w^{-N_{0}}\right|<\varepsilon / 2$ при $|w|=1$ и $\left|F_{1}(w)\right|<1+\varepsilon / 2$ при $|w| \leqslant 1$.

2) $m>k$ :

$$
F_{2}(w)=\sum_{k=k_{1}}^{k_{2}} \varepsilon_{k} \alpha_{k} w^{\alpha_{k}-N_{0}} \sum_{m=k+1}^{\infty} \frac{1}{m \alpha_{m}} \bar{w}^{\alpha_{m}} .
$$

Из (3.3) следует, что, увеличивая $k_{1}$, можно сделать $\left|F_{2}(w)\right|$ при $|w| \leqslant 1$ как угодно малым.

3) $m<k$ :

$$
F_{3}(w)=\sum_{k=k_{1}}^{k_{2}} \varepsilon_{k} \alpha_{k} w^{\alpha_{k}-N_{0}}\left(\bar{w}+\sum_{m=k_{0}}^{k-1} \frac{1}{m \alpha_{m}} \bar{w}^{\alpha_{m}}\right) .
$$


Рассмотрим многочлен

$$
\Phi_{2}(w)=\sum_{k=k_{1}}^{k_{2}} \varepsilon_{k} \alpha_{k} w^{\alpha_{k}-N_{0}}\left(w^{-1}+\sum_{m=k_{0}}^{k-1} \frac{1}{m \alpha_{m}} w^{-\alpha_{m}}\right)
$$

(так как $\alpha_{k+1}-\alpha_{k}-N_{0}>(1 / 2) \alpha_{k+1}$, степени $w$ положительны). В силу выбора $\Phi_{2}$ при $|w|=1$ имеет место равенство $F_{3}(w)=\Phi_{2}(w)$ и, следовательно, оценка 1) установлена. Оценим $\left|F_{3}(w)-\Phi_{2}(w)\right|$ при $|w|<1$. Учитывая неравенство $1-(w \bar{w})^{\alpha_{m}} \leqslant \alpha_{m}(1-w \bar{w})$, получим

$$
\begin{aligned}
\left|F_{3}(w)-\Phi_{2}(w)\right| & \leqslant(1-w \bar{w}) \sum_{k=k_{1}}^{k_{2}} \varepsilon_{k} \alpha_{k}|w|^{\alpha_{k} / 2}\left(1+\sum_{m=1}^{k-1} \frac{1}{m}\right) \\
& \leqslant A \max _{k \in\left[k_{1}, k_{2}\right]}\left(\varepsilon_{k} \log k\right)(1-|w|) \sum_{k=1}^{\infty} \alpha_{k}|w|^{\alpha_{k} / 2} .
\end{aligned}
$$

Так как имеет место условие 1) из (3.4), то для того чтобы завершить доказательство оценки 2) и доказать оценку 3) (см. (3.5)), надо показать, что при $r<1$

$$
(1-r) \sum_{k=1}^{\infty} \alpha_{k} r^{\alpha_{k} / 2}<A
$$

Действительно, достаточно рассмотреть случай $r \geqslant 1 / 2$. Пусть

$$
N(r)=\left[\log _{2}\left(\frac{1}{1-r}\right)\right]
$$

Тогда

$$
(1-r) \sum_{k=1}^{\infty} \alpha_{k} r^{\alpha_{k} / 2} \leqslant A_{1}(1-r) \sum_{k=1}^{\infty} 2^{k} r^{2^{k}} \leqslant A_{1} \sum_{k=1}^{N(r)} 2^{k-N(r)}+A_{2} \sum_{k=1}^{\infty} 2^{k} e^{-2^{k}}<A .
$$

Лемма доказана.

Для построения примера 2 используется следующее утверждение, обобщающее лемму 3.1.

Лемма 3.2. Пусть $f$ - произвольная непрерьвная функиия на $\partial K, M=$ $\max |f(w)| н$ на $\partial K$. Тогда для любого $\varepsilon>0$ и любой функции $h$ из леммы 3.1 существует функция $F=\Phi_{1} \bar{h}-\Phi_{2}\left(\right.$ дде $\Phi_{1}(w)$ и $\Phi_{2}(w)$ - многочлены $)$ такая, чmo:

1) $|F(w)-f(w)| \leqslant \varepsilon M n p u|w|=1$;

2) $|F(w)| \leqslant(1+\varepsilon) M$ npu $|w| \leqslant 1$;

3) $\max \left(\left|\Phi_{1}(w)\right|,\left|\Phi_{2}(w)\right|\right) \leqslant \varepsilon M(1-|w|)^{-1}$ npu $|w|<1$;

4) $|F(w)| \leqslant \varepsilon M n p u|w|<1-\varepsilon$.

ДокАЗАТЕЛьСтво. Не нарушая общности рассуждений, считаем, что $M \geqslant 1$. Для произвольного $N \in \mathbb{N}$ рассмотрим многочлен следующего вида:

$$
\Phi_{1}=\sum_{m=1}^{N} c_{m} \Phi_{1, m}, \quad \text { где } \Phi_{1, m}(w)=\sum_{k=k_{1, m}}^{k_{2, m}} \varepsilon_{k} \alpha_{k} w^{\alpha_{k}-N_{m}}
$$


$\Phi_{1, m}$ - многочлены из $(3.5)$, причем $k_{1, m+1}>k_{2, m}, N_{m} \in \mathbb{Z}$. Как следует из $(3.7)$, в $K$ имеет место оценка:

$$
\left|\Phi_{1}(w)\right| \leqslant A(1-|w|)^{-1} \max _{m}\left|c_{m}\right| \varepsilon_{k_{1,1}} .
$$

В силу леммы 3.1 для $m=1, \ldots, N$ и любого $\varepsilon>0$ существуют числа $\varepsilon_{m}^{\prime}$ и $\varepsilon_{m}^{\prime \prime}$ такие, что $\varepsilon_{m}^{\prime \prime}<\varepsilon_{m}^{\prime}<\varepsilon$ и $\varepsilon_{m+1}^{\prime}<\varepsilon_{m}^{\prime \prime}$, и многочлены $\Phi_{2, m}(w)$ из $(3.6)$ такие, что для функций $F_{m}=\Phi_{1, m} \bar{h}-\Phi_{2, m}$ будут выполнены неравенства $\left|c_{m} F_{m}(w)\right|<\varepsilon / 2^{m+1}$ при $|w|<1-\varepsilon_{m}^{\prime}$ и $\left|c_{m}\right|\left|F_{m}\left(r e^{i \varphi}\right)-e^{-i N_{m} \varphi}\right|<\varepsilon / 2^{m+1}$ при $|w|>1-\varepsilon_{m}^{\prime \prime}$.

Тогда для функции $F=\sum_{m=1}^{N} c_{m} F_{m}$ получим, что $\left|F\left(e^{i \varphi}\right)-P\left(e^{i \varphi}\right)\right|<\varepsilon$, где $P\left(e^{i \varphi}\right)=\sum_{m=1}^{N} c_{m} e^{-i N_{m} \varphi}$, а при $|w|<1$ :

$$
|F(w)|<\max _{s=1, \ldots, N ; \varphi \in[0,2 \pi)}\left|\sum_{m=1}^{s} c_{m} e^{-i N_{m} \varphi}\right|+\max _{m}\left|c_{m}\right|+\varepsilon .
$$

Заметим, что числа $N_{m}$ не обязательно различны, поэтому, изменяя $c_{m}$, можно записать тригонометрический многочлен $P$ в виде $\sum_{m=1}^{N} c_{m} e^{-i N_{m} \varphi}$ разными способами. В частности, можно взять $m$ достаточно большим и так выбрать $c_{m}$, “измельчая" коэффициенты Фурье $P$, что будет выполнена оценка

$$
|F(w)|<\max _{\varphi \in[0,2 \pi)}\left|P\left(e^{i \varphi}\right)\right|+\varepsilon .
$$

Равномерно приближая исходную функцию $f$ тригонометрическими многочленами, получим оценки 1) и 2). Оценки 3) и 4) следуют из (3.8), если $k_{1,1}$ достаточно велико. Лемма доказана.

ТЕОРема 3. Для любой функиии $f$, непрерывной на $\partial K$, существует функция $F_{f}=\Phi_{1} \bar{h}-\Phi_{2}\left(\Phi_{1} u \Phi_{2}\right.$ - аналитичны в $\left.K\right)$, непрерывная при $|w| \leqslant 1 u$ такая, что $F_{f}=f$ на $\partial K$.

ДокАЗАТЕЛЬСтво. Пусть $M=\max |f(w)|$ на $\partial K$. Определим следующие последовательности функций $\left\{f_{(n)}\right\}$ и $\left\{F_{(n)}=\Phi_{1,(n)} \bar{h}-\Phi_{2,(n)}\right\}$.

Пусть

$$
f_{(0)}=f, \quad f_{(n)}\left(e^{i \varphi}\right)=f_{(n-1)}\left(e^{i \varphi}\right)-F_{(n)}\left(e^{i \varphi}\right),
$$

где $F_{(n)}$ - функция из леммы 3.2 , построенная по $f_{(n-1)}$ с $\varepsilon_{(n)}=1 / 2^{n}$. Следовательно, всюду на $\partial K$

$$
\left|f\left(e^{i \varphi}\right)-\sum_{m=1}^{n} F_{(n)}\left(e^{i \varphi}\right)\right|=\left|f_{(n-1)}\left(e^{i \varphi}\right)-F_{(n)}\left(e^{i \varphi}\right)\right|=\left|f_{n}\left(e^{i \varphi}\right)\right| \leqslant \frac{M}{2^{n}} .
$$

Так как $\left|f_{(n-1)}\left(e^{i \varphi}\right)\right| \leqslant M / 2^{n-1}$, то в силу условий 2$\left.), 3\right)$ леммы 3.2 можем считать, что $\left|F_{(n)}(w)\right| \leqslant M / 2^{n-2}$ при $|w| \leqslant 1$ и $\max \left(\left|\Phi_{1,(n)}(w)\right|,\left|\Phi_{2,(n)}(w)\right|\right) \leqslant$ $\left(M / 2^{n-1}\right)(1-|w|)^{-1}$ при $|w|<1$, поэтому ряды $\sum_{n=1}^{\infty} \Phi_{1,(n)}(w)$ и $\sum_{n=1}^{\infty} \Phi_{2,(n)}(w)$ сходятся в $K$ абсолютно и равномерно.

Пусть

$$
F_{f}=\sum_{n=1}^{\infty} F_{(n)}=\sum_{n=1}^{\infty} \Phi_{1,(n)} \bar{h}-\sum_{n=1}^{\infty} \Phi_{2,(n)}=\Phi_{1} \bar{h}-\Phi_{2} .
$$

В силу (3.9) $F_{f}\left(e^{i \varphi}\right) \equiv f\left(e^{i \varphi}\right)$. Теорема доказана. 
Построение примера 2 на этом, конечно, не завершено. Проблема состоит в том, что функция $h$ из леммы 3.1 не может быть однолистной в $K$, так как условия, указанные в замечаниях 1) и 3 ) перед леммой 3.1 , несовместны.

\section{§4. Пример 2: конструкция области $G$}

Пусть $G$ - жорданова область со спрямляемой границей $\partial G$. Прежде всего в леммах 4.1 и 4.2 получим условия, равносильные условию $S_{2}(\partial G, G)=C(\partial G)$ и удобные для проверки, затем вернемся к построению примера. Заметим, что теорема 3 (как следствие леммы 3.2) является частным случаем следующего утверждения функционального анализа (см., например, [6; лемма 4.13]).

УтВЕРЖДЕНИЕ. Пусть $U$ и $V$ - открытые единичные шары в банаховых пространствах $X$ и $Y, T$-ограниченный линейный оператор $T: X \rightarrow Y$. Если для некоторого $\lambda>0$ образ шара $\lambda U$ плотен в $V$, то он содержит $V$.

Применим указанное утверждение в следующей ситуации: $X=A_{2}(\bar{G})$ (полнота $X$ следует из того, что в силу (1.2) сходимость бианалитических функций в $C(\bar{G})$ влечет равномерную сходимость их аналитических компонент в $G)$, $Y=C(\partial G), T$ - оператор сужения на $\partial G$ функции, непрерывной на $\bar{G}$. Таким образом, для установления равенства $S_{2}(\partial G, G)=C(\partial G)$ достаточно убедиться в том, что существует $\lambda>0$, при котором множество $T(\lambda U)$ плотно в единичном шаре из $C(\partial G)$. В противном случае, так как множество $\overline{T(\lambda U)}$ замкнуто и выпукло, по теореме об отделимости выпуклых множеств существует линейный непрерывный функционал пространства $C(\partial G)$, отделяющий $\overline{T(\lambda U)}$ от некоторой функции $f \in C(\partial G),\|f\|_{\mathrm{L}_{\infty}} \leqslant 1$. Поэтому для установления равенства $S_{2}(\partial G, G)=C(\partial G)$ достаточно показать, что таких функционала и функции $f$ не существует и, таким образом, имеет место следующее утверждение.

Лемма 4.1. Условие $S_{2}(\partial G, G)=C(\partial G)$ равносильно следующему.

Существует $\lambda>0$, при котором для любых функиии $f \in C(\partial G)$ такой, что $\|f\|_{\mathrm{L}_{\infty}}(\partial G) \leqslant 1$, конечной комплексной борелевской меры $\mu$ на $\partial G$ с полной вариацией $\|\mu\|(\partial G)=1 u \varepsilon>0$ существует функиия $F=F(f, \mu, \varepsilon) \in A_{2}(\bar{G})$ такая, что $|F(z)|<\lambda$ в $G$ и имеет место оценка

$$
\left|\int_{\partial G}(f(z)-F(z)) d \mu_{z}\right|<\varepsilon .
$$

ЗАмечАниЕ. В лемме 4.1 существенно, что функции $F$ равномерно ограничены в $G$. В противном случае получим более слабое утверждение - плотность $S_{2}(\partial G, G)$ в $C(\partial G)$. Этот факт имеет место тогда и только тогда, когда $\partial G$ не является неванлинновским контуром [8; теорема 1]; простейший пример контура, не являющегося неванлинновским, - эллипс, отличный от окружности.

С помощью теоремы Рудина-Карлесона [9], [10] и леммы 4.1 получим следующее утверждение, которое будем использовать для построения примера 2.

ЛЕмма 4.2. Условие $S_{2}(\partial G, G)=C(\partial G)$ равносильно следующему. 
Существует возрастающая последовательность $\left\{E_{n}\right\}, n=1,2, \ldots$, замкнутых подмножеств $\partial G$ таких, что длина множества $\Omega=\partial G \backslash \bigcup_{n} E_{n}$ равна нулю, и при этом для любъх функиии $f \in C(\partial G)$ такой, что $\|f\|_{\mathrm{L}_{\infty}}(\partial G) \leqslant 1$, множества $E_{n}$ и $\varepsilon>0$ существует функиия $\widetilde{F}=\widetilde{F}\left(f, E_{n}, \varepsilon\right) \in A_{2}(\bar{G})$ такая, что $|\widetilde{F}(z)| \leqslant 2$ в $G$ u $|f(z)-\widetilde{F}(z)|<\varepsilon$ на $E_{n}$.

ДокАЗАтЕльство. Достаточно показать, что в условиях леммы 4.2 выполнена оценка (4.1). Пусть $\mu$ - мера из леммы 4.1, $\|\mu\|(\partial G)=1$. Зададим произвольное $\varepsilon>0$. Возьмем $\Omega^{\prime}-$ замкнутое подмножество $\Omega$ такое, что $\|\mu\|\left(\Omega \backslash \Omega^{\prime}\right)<\varepsilon / 16$. Зафиксируем номер $n$ такой, что $\|\mu\|\left(\Omega_{n} \backslash \Omega^{\prime}\right)<\varepsilon / 8$. Пусть $\widetilde{F} \in A_{2}(\bar{G})-$ функция такая, что $|\widetilde{F}(z)| \leqslant 2$ в $G$ и $|f(z)-\widetilde{F}(z)|<\varepsilon / 8$ на $E_{n}$.

Рассмотрим функцию $r=f-\widetilde{F}$. Это непрерывная функция на $\partial G$ и $|r(z)| \leqslant 3$ в $\bar{G}$. Напомним, что $\Omega^{\prime}-$ замкнутое множество длины нуль. По теореме Рудина-Карлесона [9], [10] существуют функции $\Psi_{r}$ и $\Psi_{1}$, аналитические в $G$ и непрерывные в $\bar{G}$, такие, что на $\Omega^{\prime}$ имеют место равенства $\Psi_{r}(z) \equiv r(z)$ и $\Psi_{1}(z) \equiv 1$, а на множестве $\bar{G} \backslash \Omega^{\prime}$ выполняются неравенства $\left|\Psi_{r}(z)\right|<3$ и $\left|\Psi_{1}(z)\right|<1$ соответственно. В частности, для любого $N \in \mathbb{N}$ на $\Omega^{\prime}$ имеем $\Psi_{r}(z)\left(\Psi_{1}(z)\right)^{N} \equiv r(z)$.

Рассмотрим бианалитическую в $G$ и непрерывную в $\bar{G}$ функцию $F=\widetilde{F}+$ $\left(\Psi_{1}\right)^{N} \Psi_{r}$. Так как на $\Omega^{\prime}$ имеет место равенство $f(z)=F(z)$, то

$$
\begin{gathered}
\left|\int_{\partial G}(f(z)-F(z)) d \mu_{z}\right|=\left|\int_{\partial G \backslash \Omega^{\prime}}(f(z)-F(z)) d \mu_{z}\right| \\
\leqslant \int_{E_{n}}|f(z)-\widetilde{F}(z)| d\left|\mu_{z}\right|+\int_{\Omega_{n} \backslash \Omega^{\prime}}|f(z)-F(z)| d\left|\mu_{z}\right| \\
\quad+\int_{E_{n}}\left|\Psi_{r}(z)\left(\Psi_{1}(z)\right)^{N}\right| d\left|\mu_{z}\right|<\frac{\varepsilon}{8}+\frac{6 \varepsilon}{8}+\frac{\varepsilon}{8}=\varepsilon,
\end{gathered}
$$

если $N$ достаточно велико. Оценка (4.1) установлена. Лемма доказана.

Таким образом, пример 2 сводится к построению области $G$, граница которой содержит последовательность замкнутых множеств $\left\{E_{n}\right\}$, удовлетворяющих условиям леммы 4.2 .

Сначала укажем общую схему конструкции и введем основные обозначения. Область $G$ строится как образ некоторой области $D \subset K$ при отображении соответствующей аналитической и однолистной в $D$ функцией $H$, причем для $D$ и $H$ выполнены условия следующей леммы, а $\delta>0$ при построении можно сделать как угодно малым.

Лемма 4.3. Пусть D - жорданова область с липшицевой гранищей $\partial D$, $H$ - бункция, аналитическая в $D$, причем функиия $H^{\prime}$ непрерывна в $\bar{D}$. Если для $\delta<1 / 2$ в $D$ выполняется неравенство $\left|H^{\prime}(w)-1\right|<\delta$, а для произвольных граничных точек $w_{1}$ и $w_{2}$ длина кратчайшего пути внутри $D$, соединяющего $w_{1}$ и $w_{2}$, не превосходит $2\left|w_{2}-w_{1}\right|$, то функиия $H$ однолистно отображает $D$ на жорданову область со спрямляемой гранищей. 
Действительно,

$$
\left|H\left(w_{2}\right)-H\left(w_{1}\right)\right|=\left|\int_{w_{1}}^{w_{2}} H^{\prime}(w) d w\right| \geqslant\left|w_{2}-w_{1}\right|-2 \delta\left|w_{2}-w_{1}\right|>0,
$$

поэтому $H$ взаимно однозначно отображает $\partial D$ на замкнутую жорданову спрямляемую кривую и, следовательно (см., например, [13; гл. 5, §1]), $H$ однолистна в $D$. Если еще уменьшить $\delta$, образ $\partial D$ будет липшицевой кривой.

Чтобы добиться выполнения условий леммы 4.2, будем строить область $D$ так, чтобы существовала возрастающая последовательность $\left\{E_{n}^{\prime}\right\}, n=1,2, \ldots$, замкнутых подмножеств $\partial D$ (длина множества $\partial D \backslash\left(\bigcup_{n} E_{n}^{\prime}\right)$ равна нулю), обладающая следующим свойством.

Для любых функции $f$, непрерывной на $\partial D$ такой, что $\|f\|_{\mathrm{L}_{\infty}}(\partial G) \leqslant 1$, множества $E_{n}^{\prime}$ и $\varepsilon>0$ существует функция $F=\Phi_{1} \bar{H}-\Phi_{2}$ (где $\Phi_{1}$ и $\Phi_{2}$ аналитичны в $D$, а $H$ удовлетворяет условиям леммы 4.3$)$ такая, что $|F(w)| \leqslant 2$ в $D$ и $|f(w)-F(w)|<\varepsilon$ на $E_{n}^{\prime}$.

Тогда, очевидно, в качестве искомой области $G$ можно взять образ $D$ при отображении функцией $H$, а в качестве множеств $E_{n}$ леммы 4.2 - образы $E_{n}^{\prime}$.

Область $D$ строится как ядро убывающей последовательности вложенных жордановых областей $K=K_{0} \supset K_{1} \supset K_{2} \supset \cdots \supset D$. Выполнение условий, налагаемых на область $D$ леммой 4.3 , будет достигнуто в силу того, что все области $K_{n}$ будут звездными относительно $w=0$, и их границы в полярных координатах можно будет задать липшицевыми функциями $\rho_{n}(\varphi)$, где

$$
\rho_{0}(\varphi) \equiv 1, \quad \frac{9}{10}<\rho_{n}(\varphi) \leqslant 1, \quad\left|\rho_{n-1}^{\prime}(\varphi)-\rho_{n}^{\prime}(\varphi)\right|<\left(\frac{1}{10}\right)^{n}, \quad n \geqslant 1 .
$$

Рассмотрим строение границ областей $K_{n}$ подробней. Граница каждой области $K_{n}$ при $n \geqslant 1$ состоит из замкнутого множества $E_{n}^{\prime}$ и семейства интервалов, дополнительных к $E_{n}^{\prime}$, длина открытого множества $\partial K_{n} \backslash E_{n}^{\prime}$ меньше $(1 / 2)^{n}$. Каждый дополнительный интервал либо прямолинеен, либо является объединением двух прямолинейных полуинтервалов с общей точкой. При этом выполнены условия $E_{n-1}^{\prime} \subset E_{n}^{\prime}$, а именно, $E_{n}^{\prime}=E_{n-1}^{\prime} \cup \Delta E_{n}^{\prime}$, где $\Delta E_{n}^{\prime}-$ замкнутое множество, не пересекающее $E_{n-1}^{\prime}$ (причем $E_{0}^{\prime}=\varnothing$ и $E_{1}^{\prime}=\Delta E_{1}^{\prime}$ ). Таким образом, $\partial D$ содержит возрастающую последовательность замкнутых множеств $E_{n}^{\prime}$, и длина множества $\partial D \backslash\left(\bigcup_{n} E_{n}^{\prime}\right)$ равна нулю.

Рассмотрим строение множеств $\Delta E_{n}^{\prime}$. Каждое из них имеет вид $\Delta E_{n}^{\prime}=$ $\bigcup_{s=1}^{N_{n}} \Delta E_{n, s}^{\prime}$ (замкнутые множества $\Delta E_{n, s}^{\prime}$ попарно не пересекаются).

Множества $\Delta E_{n, s}^{\prime}$ имеют специальное строение, для описания которого понадобятся круги $B_{n, s}$ и жордановы области с дважды гладкой границей $\widetilde{B_{n, s}} \subset B_{n, s}$ (в случае $n=1$ возьмем $N_{1}=1$, под $B_{1,1}=\widetilde{B_{1,1}}$ будем понимать "несобственный" круг - внешность $\left.\bar{K}, \Delta E_{1,1}^{\prime} \subset \partial K\right)$. При этом выполнены следующие условия.

1) Некоторая окрестность замыкания области $\widetilde{B_{n, s}}$ не пересекает круг $B_{n_{1}, s_{1}}$, если $(n, s) \neq\left(n_{1}, s_{1}\right)$ (сами круги $B_{n, s}$ могут попарно пересекаться).

2) Все $B_{n, s}$ расположены вне $\overline{K_{n}}$ (и, следовательно, вне $\overline{K_{m}}$ при $m>n$ и вне $\bar{D})$. 
3) При $n \leqslant m$ имеют место равенства

$$
\Delta E_{n, s}^{\prime}=\partial B_{n, s} \cap \overline{K_{m}}=\partial \widetilde{B_{n, s}} \cap \overline{K_{m}} .
$$

4) Как вытекает из 2) и 3), множество

$$
\partial K_{n} \backslash E_{n}^{\prime}=\partial K_{n} \backslash\left(\bigcup_{m=1}^{n} \bigcup_{s=1}^{N_{m}} \Delta E_{m, s}^{\prime}\right)
$$

расположено во внешности $\bigcup_{m=1}^{n} \bigcup_{s=1}^{N_{m}} \overline{B_{m, s}}$.

Функция $H$ будет построена в виде суммы $H=h_{1,1}+\sum_{n=2}^{\infty} \sum_{s=1}^{N_{n}} h_{n, s}$, где каждая функция $h_{n, s}$ удовлетворяет следующим условиям.

1) Функция $h_{n, s}$ непрерывна вне $\widetilde{B_{n, s}}$ и аналитична вне замыкания $\widetilde{B_{n, s}}$ (и, следовательно, аналитична вне $\left.\overline{B_{n, s}}\right)$, причем $h_{n, s}(\infty)=0, n>1$. Так как области $\widetilde{B_{n, s}}$ расположены вне $\overline{K_{n}}$, то функция $h_{n, s}$ аналитична в $K_{n}$ и, следовательно, в $K_{m}$ при $m>n$ и в $D$; в силу строения множества $\partial K_{n} \backslash E_{n}^{\prime}$ (условие 4$)$ ) функция $h_{m, s}$ при $m \leqslant n$ аналитична в окрестности каждой точки множества $\partial K_{n} \backslash E_{n}^{\prime}$.

2) Функция $h_{n, s}$ непрерывно дифференцируема в замкнутой области $\overline{K_{n}}$, при этом $\left|1-h_{1,1}^{\prime}(w)\right|<\delta_{1}$ в $\overline{K_{1}}$, а при $n>1$ выполнены оценки $\left|h_{n, s}^{\prime}(w)\right|<\delta_{n} / N_{n}$ в $\overline{K_{n}}$, где $\sum_{n=1}^{\infty} \delta_{n}<\delta$, а $\delta_{n}>0$ и $\delta<1 / 2$ - фиксированные до построения произвольно малые положительные постоянные. Следовательно, те же оценки производных имеют место и в $\overline{K_{m}}, m>n$, и в $\bar{D}$.

Рассмотрим конструкцию в деталях. Пусть сначала $n=1$.

Функция $h_{1,1}$ и область $K_{1}$ строятся так: $h_{1,1}=h_{k_{0}}$ из (3.2) при достаточно большом $k_{0}$ (значение $k_{0}$ зафиксируем несколько позже), а $K_{1}$ получается из $K$ с помощью конструкции Лузина-Привалова (см. [2; гл. 4, §2, п. 2.4], $[11 ;$ гл. $3, \S \mathrm{D}])$. Действительно, хотя функция $h_{k_{0}}^{\prime}$ и не ограничена в $K$, но $\mathrm{L}_{2}$-норма функции $h_{k_{0}}^{\prime}-1$ на $\partial K$ может быть сделана как угодно малой вместе с $\sum_{k=k_{0}}^{\infty} 1 / k^{2}$, то же касается и $\mathrm{L}_{2}$-нормы, соответствующей некасательной максимальной функции (см., например, [14; гл. $1, \S 5]$ ). Поэтому для достаточно большого $k_{0}=k_{0}\left(\delta_{1}\right)$ существует область $K_{1}$, полученная выбрасыванием из $K$ счетного семейства попарно не пересекающихся равнобедренных треугольников с основаниями на $\partial K$, такая, что функция $h_{k_{0}}^{\prime}$ будет непрерывна в $\overline{K_{1}}$, причем в $\overline{K_{1}}$ будет выполнена оценка $\left|h_{k_{0}}^{\prime}(w)-1\right|<\delta_{1}$. При этом угол при основании каждого выброшенного треугольника и сумма периметров всех треугольников будут меньше 1/20 (соответствующие оценки на этом и следующих шагах гарантируют липшицевость $\partial D$ и равенство нулю длины множества $\left.\partial D \backslash\left(\bigcup_{n} E_{n}^{\prime}\right)\right)$. В качестве $\Delta E_{1}^{\prime}$ возьмем $\partial K_{1} \cap \partial K$; нетрудно убедиться, что $\Delta E_{1}^{\prime}$ и $h_{1,1}$ удовлетворяют сформулированным выше условиям, налагаемым на $\Delta E_{n, s}^{\prime}$ и $h_{n, s}$.

Рассмотрим переход от $K_{n}$ к $K_{n+1}$, который существенно сложнее, чем построение $K_{1}$. Сначала строится "промежуточная" область $\widetilde{K_{n+1}} \subset K_{n}\left(\widetilde{K_{1}}=K\right.$ при $n=1)$. Затем строятся функции $h_{n+1, s}$ и вспомогательные функции $h_{k, s}^{k_{1}, s_{1}}$, $(k, s) \neq\left(k_{1}, s_{1}\right), k \leqslant n+1, k_{1} \leqslant n+1$ (те из них, которые не были построены на 
предыдущих шагах), и с помощью конструкции Лузина-Привалова строится область $K_{n+1} \subset \widehat{K_{n+1}}$.

Предварительно поясним, с какой целью вводятся вспомогательные функции и какой вид они имеют. Напомним, что для любого $\varepsilon>0$ существуют многочлены $\Phi_{1,(1,1)}(w)$ и $\Phi_{2,(1,1)}(w)$ такие, что функция $\Phi_{1,(1,1)} \overline{h_{1,1}}-\Phi_{2,(1,1)}$ удовлетворяет условиям леммы 3.2. Это позволяет равномерно приближать на $\Delta E_{1}^{\prime}$ произвольную непрерывную функцию $f$ функциями вида $\Phi_{1,(1,1)} \overline{h_{1,1}}-\Phi_{2,(1,1)}$ с оценками 1)-4) леммы 3.2. Однако этого недостаточно, так как нужно приближать $f$ функциями вида $\Phi_{1} \bar{H}-\Phi_{2}$, где $H=h_{1,1}+\sum_{n=2}^{\infty} \sum_{s=1}^{N_{n}} h_{n, s}$, причем функции $\Phi_{1,(1,1)} \overline{h_{n, s}}$ при $n>1$ вблизи $\partial K$ могут расти как угодно быстро. Чтобы скомпенсировать этот рост, вместе с $h_{n, s}$ строим вспомогательные функции $h_{n, s}^{n_{1}, s_{1}}$, где $(n, s) \neq\left(n_{1}, s_{1}\right)$, каждая из этих функций аналитична вне компактного подмножества круга $B_{n_{1}, s_{1}}$ (напомним, что круг $B_{n_{1}, s_{1}}$ расположен вне $\overline{K_{n_{1}}}$ и окрестность замыкания $\widetilde{B_{n, s}}$ не пересекает круг $B_{n_{1}, s_{1}}$, если $(n, s) \neq\left(n_{1}, s_{1}\right)$; добиться выполнения этих условий, приведенных выше, - одна из задач при построении $\left.K_{n+1}\right)$.

Функции $h_{n, s}^{n_{1}, s_{1}}$ строятся с помощью инверсий относительно окружностей $\partial B_{n_{1}, s_{1}}$. Так, при $n>1$ возьмем $h_{n, s}^{1,1}(w)=\overline{h_{n, s}(1 / \bar{w})}$. Очевидно, что на окружности $\partial K$ имеет место равенство $\overline{h_{n, s}(w)}=h_{n, s}^{1,1}(w)$. Аналогично, пусть $B=B_{n_{1}, s_{1}}-$ круг с центром $w_{0}$ радиуса $r$. Тогда возьмем

$$
h_{n, s}^{n_{1}, s_{1}}(w)=\overline{h_{n, s}\left(w_{0}+\frac{r^{2}}{\overline{w-w_{0}}}\right)}
$$

(и на $\partial B_{n_{1}, s_{1}}$ получим равенство $\left.h_{n, s}^{n_{1}, s_{1}}(w)=\overline{h_{n, s}(w)}\right)$. Основная задача при построении вспомогательных функций - добиться выполнения оценок (4.7), уточняющих указанные равенства.

Вернемся к построению области $K_{n+1}$.

Пусть область $K_{n}, n \geqslant 1$, построена, $l_{n}$ - длина открытого подмножества $\partial K_{n}$, дополнительного к $E_{n}^{\prime}, l_{n}<(1 / 2)^{n}$. Напомним, что каждый дополнительный интервал для $E_{n}^{\prime}$ либо прямолинеен, либо является объединением двух прямолинейных полуинтервалов с общей точкой. Возьмем конечное число отрезков $e_{n+1, s}$, лежащих на $\partial K_{n} \backslash E_{n}^{\prime}$, так, чтобы все отрезки имели равную длину, отрезки $1.01 e_{n+1, s}$ попарно не пересекались и не пересекали $E_{n}^{\prime}$, при этом сумма длин всех $e_{n+1, s}$ была больше $0.9 l_{n}$. Для каждого из отрезков $e_{n+1, s}$ проведем окружность с центром $w_{n+1, s}$ вне $\overline{K_{n}}$, проходящую через концы отрезка (радиусы окружностей для всех $e_{n+1, s}$ равны; обозначим их через $\left.r_{n+1}\right)$, соответствующие круги обозначим через $B_{n+1, s}$.

Будем считать отрезки и круги настолько малыми, что выполняются следующие условия.

1) Величина угла внутри $K_{n}$ между окружностью $\partial B_{n+1, s}$ и отрезком $e_{n+1, s}$ меньше $(1 / 20)^{n+1}$;

2 ) Круги $2 B_{n+1, s}$ не пересекают $\overline{B_{m, s}}, m \leqslant n$ (в частности, не пересекают $\partial K)$. Это можно сделать в силу строения $\partial K_{n} \backslash E_{n}^{\prime}($ условие 4$\left.)\right)$. 
3) На $2 B_{n+1, s}$ имеют место оценки $\left|1-h_{1,1}^{\prime}(w)\right|<2 \delta_{1}$ и $\left|h_{m, s}^{\prime}(w)\right|<2 \delta_{m} / N_{m}$ при $2 \leqslant m \leqslant n$ (с несколько большими постоянными, чем в $\overline{K_{n}}$ ). Это можно сделать, так как функции $h_{m, s}, m \leqslant n$, аналитичны в окрестности каждой точки множества $\partial K_{n} \backslash E_{n}^{\prime}$ (условие 1$)$, которому удовлетворяют функции $h_{m, s}$ ).

Таким образом, существует конечное число отрезков $e_{n+1, s}$, удовлетворяющих указанным условиям. Это число обозначим через $N_{n+1}$. Зафиксируем "промежуточную" область $\widetilde{K_{n+1}}=K_{n} \backslash \bigcup_{s=1}^{N_{n+1}} \overline{B_{n+1, s}}$.

Построим области $\widetilde{B_{n+1, s}}$, функции $h_{n+1, s}$, множества $\Delta E_{n+1, s}^{\prime}$ и область $K_{n+1}$.

Заметим, что круги $B_{n+1, s}$ могут попарно пересекаться, но в силу построения попарно не пересекаются некоторые окрестности сегментов $B_{n+1, s} \cap K_{n}$, каждый из которых ограничен отрезком $e_{n+1, s}$ и дугой окружности $\Gamma_{n+1, s}=$ $\partial B_{n+1, s} \cap \partial \widetilde{K_{n+1}}$. Поэтому возьмем жордановы области $\widetilde{B_{n+1, s}} \subset B_{n+1, s}$ с дважды гладкой границей такие, что $\partial \widetilde{B_{n+1, s}} \cap \partial \widetilde{K_{n+1}}=\Gamma_{n+1, s}$, при этом для каждого $s$ некоторая окрестность $U_{n+1, s}$ замыкания области $\overparen{B_{n+1, s}}$ не пересекается с кругом $B_{n+1, s_{1}}$, если $s \neq s_{1}$.

Пусть $\chi_{n+1, s}$ - аналитическая функция, однолистно отображающая внешность $\widehat{B_{n+1}, s}$ на $K$ (считаем, что $\left.\chi_{n+1, s}(\infty)=0\right)$. В силу достаточной гладкости $\partial \widetilde{B_{n+1, s}}$ функция $\chi_{n+1, s}$ непрерывно дифференцируема вне $\widetilde{B_{n+1, s}}$ и $0<$ $\left|\chi_{n+1, s}^{\prime}(w)\right|<\infty$ на произвольном ограниченном подмножестве дополнения K $\overparen{B_{n+1, s}}$.

Пусть $h(w)=h_{k_{0}}(w)-w-$ функция из леммы 3.2 при произвольном $k_{0}$. Напомним, что

$$
h(w)=\sum_{k=k_{0}}^{\infty} \frac{1}{k \alpha_{k}} w^{\alpha_{k}}, \quad \frac{\alpha_{k+1}}{\alpha_{k}} \geqslant 2,
$$

нужное значение $k_{0}$ зафиксируем несколько позже.

Возьмем $h_{n+1, s}(w)=h\left(\chi_{n+1, s}(w)\right)$. Воспользовавшись леммой 3.2 и свойствами функции $\chi_{n+1, s}$, получим, что для любой функции $f$, непрерывной на $\partial \widetilde{B_{n+1, s}}$, такой, что $\|f\|_{\mathrm{L}_{\infty}} \leqslant 1$, и для любого $\varepsilon_{n+1, s}>0$ существуют функции $\Phi_{1,(n+1, s)}=\Phi_{1}\left(\chi_{n+1, s}\right)$ и $\Phi_{2,(n+1, s)}=\Phi_{2}\left(\chi_{n+1, s}\right)$ (где $\Phi_{1}$ и $\Phi_{2}-$ многочлены из леммы 3.2), аналитические вне замыкания $\widehat{B_{n+1, s}}$ и равные нулю на бесконечности, такие, что для соответствующей функции

$$
F_{n+1, s}=\Phi_{1,(n+1, s)} \overline{h_{n+1, s}}-\Phi_{2,(n+1, s)}
$$

имеют место оценки, аналогичные оценкам 1)-4) леммы 3.2. Именно, при $m=n+1$

$$
\begin{gathered}
\left|F_{m, s}(w)-f(w)\right|<\varepsilon_{m, s} \quad \text { при } w \in \partial \widetilde{B_{m, s}}, \\
\left|F_{m, s}(w)\right|<1+\varepsilon_{m, s} \quad \text { вне } \widetilde{B_{m, s}}, \\
\max \left(\left|\Phi_{1,(m, s)}(w)\right|,\left|\Phi_{2,(m, s)}(w)\right|\right)<\varepsilon_{m, s} \max \left(1,\left(\operatorname{dist}\left(w, \widetilde{B_{m, s}}\right)\right)^{-1}\right)
\end{gathered}
$$

(это выполняется в силу леммы 3.2 и теоремы Кёбе об искажении),

$$
\left|F_{m, s}(w)\right|<\varepsilon_{m, s} \quad \text { при } \operatorname{dist}\left(w, \widetilde{B_{m, s}}\right)>\varepsilon_{m, s} .
$$


Уточним выбор $k_{0}$. Применим для всех $s$ на дугах $\Gamma_{n+1, s}$ (относительно области $\widetilde{K_{n+1}}$ ) конструкцию Лузина-Привалова так же, как и при построении $K_{1}$ из $K$. Возьмем $k_{0}$ достаточно большим таким, чтобы выполнялись следующие условия:

1) отношение суммы периметров всех выброшенных равнобедренных треугольников к сумме длин всех отрезков $e_{n+1, s}$ было меньше $(1 / 20)^{n+1}$;

2) угол при основании каждого выброшенного треугольника был меньше $(1 / 20)^{n+1}$;

3) в замыкании области $K_{n+1}$, полученной из $\widetilde{K_{n+1}}$ выбрасыванием всех указанных треугольников, выполнялись оценки $\left|h_{n+1, s}^{\prime}(w)\right|<\delta_{n+1} / N_{n+1}$;

4) при $n_{1} \leqslant n+1$ и $(n+1, s) \neq\left(n_{1}, s_{1}\right)$ для $w \notin\left(\widetilde{B_{n+1, s}} \cup B_{n_{1}, s_{1}}\right)$ и $h_{n+1, s}^{n_{1}, s_{1}}$ из (4.2) имели место оценки

$$
\left|\overline{h_{n+1, s}(w)}-h_{n+1, s}^{n_{1}, s_{1}}(w)\right| \leqslant \operatorname{dist}\left(w, \partial B_{n_{1}, s_{1}}\right) \frac{\delta_{n+1}}{N_{n+1}}
$$

(добиться выполнения указанных оценок можно, так как в силу построения область $\overparen{B_{n+1, s}}$ "хорошо отделена" от $\left.B_{n_{1}, s_{1}}\right)$.

Построение области $K_{n+1}$ завершено, при этом $\Delta E_{n+1, s}^{\prime}=\Gamma_{n+1, s} \cap \partial K_{n+1}$.

Рассмотрим теперь $\left|\overline{h_{n_{1}, s_{1}}(w)}-h_{n_{1}, s_{1}}^{n+1, s}(w)\right|$ при $n_{1}<n+1$. Напомним, что в силу (4.2)

$$
h_{n_{1}, s_{1}}^{n+1, s}(w)=\overline{h_{n_{1}, s_{1}}\left(w^{*}\right)},
$$

где $w^{*}=w_{n+1, s}+\left(r_{n+1}\right)^{2} /\left(\overline{w-w_{n+1, s}}\right)$ - точка, полученная из $w$ инверсией относительно окружности $\partial B_{n+1, s}, w^{*} \in B_{n+1, s}$.

Также напомним, что в $\overline{K_{n+1}}$ и в $2 B_{n+1, s}$ имеют место оценки $\left|h_{1,1}^{\prime}(w)\right|<$ $1+2 \delta_{1}$ и $\left|h_{n_{1}, s_{1}}^{\prime}(w)\right|<2 \delta_{n_{1}} / N_{n_{1}}$, если $1<n_{1}<n+1$; круг $2 B_{n+1, s}$ не пересекает окружностей $\partial B_{n_{1}, s_{1}}$, но пересекает $K_{n+1}$.

Пусть $w \in \overline{K_{n+1}}$. Воспользовавшись указанными оценками производных на пути, соединяющем $w$ и $w^{*}$ (поскольку для $K_{n+1}$, как и для $D$, выполнены условия леммы 4.3, то длину этого пути можно взять не большей $\left.A\left|w-w^{*}\right|\right)$, и применив теорему Лагранжа, в итоге получим следующие оценки для вспомогательных функций $h_{m_{1}, s_{1}}^{m, s}$ при $w \in \bar{D}$ :

$$
\begin{gathered}
\left|\overline{h_{1,1}(w)}-h_{1,1}^{m, s}(w)\right| \leqslant A\left(1+\delta_{1}\right)\left(\operatorname{dist}\left(w, \widetilde{B_{m, s}}\right)\right) \\
\left|\overline{h_{m_{1}, s_{1}}(w)}-h_{m_{1}, s_{1}}^{m, s}(w)\right| \leqslant A\left(\operatorname{dist}\left(w, \widetilde{B_{m, s}}\right)\right) \frac{\delta_{m_{1}}}{N_{m_{1}}}, \quad m_{1}>1 .
\end{gathered}
$$

Покажем, что область $D$ и функция $H$ - требуемые.

Возьмем для произвольного $n \in \mathbb{N}$ множество

$$
E_{n}^{\prime}=\bigcup_{m=1}^{n} \bigcup_{s=1}^{N_{m}} \Delta E_{m, s}^{\prime} .
$$

Пусть даны произвольные функция $f \in C(\partial D)$ такая, что $\|f\|_{\mathrm{L}_{\infty}}(\partial D) \leqslant 1$, и $\varepsilon=\sum_{m=1}^{n} \sum_{s=1}^{N_{m}} \varepsilon_{m, s}\left(\right.$ все $\left.\varepsilon_{m, s}>0\right)$. 
Напомним, что существуют пары функций $\Phi_{1,(m, s)}(w)$ и $\Phi_{2,(m, s)}(w), 1 \leqslant$ $m \leqslant n$, и функции $h_{m_{1}, s_{1}}^{m, s}(w), m_{1}=1,2, \ldots,\left(m_{1}, s_{1}\right) \neq(m, s)$ (все указанные функции аналитичны в $D$ и непрерывны в $\bar{D})$, такие, что для $\Phi_{1,(m, s)}$ и $\Phi_{2,(m, s)}$ выполнены оценки (4.3)-(4.6), а для $h_{m_{1}, s_{1}}^{m, s}$ выполнена оценка (4.7).

Рассмотрим функцию $F=\Phi_{1} \bar{H}-\Phi_{2}$, где

$$
\begin{gathered}
\Phi_{1}=\sum_{m=1}^{n} \sum_{s=1}^{N_{m}} \Phi_{1,(m, s)}, \quad H=\sum_{m_{1}=1}^{\infty} \sum_{s_{1}=1}^{N_{m_{1}}} h_{m_{1}, s_{1}}, \\
\Phi_{2}=\sum_{m=1}^{n} \sum_{s=1}^{N_{m}} \Phi_{2,(m, s)}+\sum_{m=1}^{n} \sum_{s=1}^{N_{m}} \sum_{\left(m_{1}, s_{1}\right) \neq(m, s)} \Phi_{1,(m, s)} h_{m_{1}, s_{1}}^{m, s} .
\end{gathered}
$$

Получим

$$
\begin{gathered}
F(w)=\Phi_{1}(w) \overline{H(w)}-\Phi_{2}(w)=\sum_{m=1}^{n} \sum_{s=1}^{N_{m}}\left(\Phi_{1,(m, s)}(w) \overline{h_{m, s}(w)}-\Phi_{2,(m, s)}(w)\right) \\
\quad+\sum_{m=1}^{n} \sum_{s=1}^{N_{m}} \Phi_{1,(m, s)}(w) \sum_{\left(m_{1}, s_{1}\right) \neq(m, s)}\left(\overline{h_{m_{1}, s_{1}}(w)}-h_{m_{1}, s_{1}}^{m, s}(w)\right) .
\end{gathered}
$$

В силу (4.5) и (4.7) будет выполнена следующая оценка в $\bar{D}$ :

$$
\begin{gathered}
\left|F(w)-\sum_{m=1}^{n} \sum_{s=1}^{N_{m}}\left(\Phi_{1,(m, s)}(w) \overline{h_{m, s}(w)}-\Phi_{2,(m, s)}(w)\right)\right| \\
<A \sum_{m=1}^{n} \sum_{s=1}^{N_{m}} \varepsilon_{m, s}\left(1+\sum_{n=1}^{\infty} \delta_{n}\right)<2 A \varepsilon
\end{gathered}
$$

Так как $\Delta E_{m, s}^{\prime} \subset \partial \widetilde{B_{m, s}}$ и области $\widetilde{B_{m, s}}, m \leqslant n$, отделены друг от друга, то можем считать, что в $(4.6)$ неравенство $\left|F_{m, s}(w)\right|<\varepsilon_{m, s}$ выполнено вне столь малой окрестности $\widetilde{B_{m, s}}$, что указанные окрестности при всех $m \leqslant n$ и соответствующих $s$ попарно не пересекаются. Отсюда в силу (4.3)-(4.4) получим $|F(w)-f(w)|<4 A \varepsilon$ на $E_{j}^{\prime}$ и при достаточно малом $\varepsilon$ соответственно $|F(w)|<2$ в $\bar{D}$. Этого достаточно (после однолистного отображения области $D$ функцией $H$ ) для выполнения условий леммы 4.2. Тем самым построение примера 2 завершено.

\section{Список литературы}

[1] М.Б. Балк, "Полианалитические функции и их обобщения", Комплексный анализ. Одна переменная - 1, Итоги науки и техн. Сер. Соврем. пробл. мат. Фундам. направления, 85, ВИНИТИ, М., 1991, 187-246; англ. пер.: М.В. Balk, "Polyanalytic functions and their generalizations", Complex analysis I, Encyclopaedia Math. Sci., 85, Springer-Verlag, Berlin, 1997, 195-253.

[2] И. И. Привалов, Граничные свойства аналитических функиий, ГИТТЛ, М.-Л., 1950. 
[3] Ph. J. Davis, The Schwarz function and its applications, The Mathematical Association of America, Washington, 1974.

[4] М. Я. Мазалов, "Пример непостоянной бианалитической функции, обращающейся в нуль всюду на нигде не аналитической границе", Матем. заметки, 62:4 (1997), 629-632; англ. пер.: M. Ya. Mazalov, "An example of a nonconstant bianalytic function vanishing everywhere on a nowhere analytic boundary", Math. Notes, 62:4 (1997), 524-526.

[5] Д. Д. Кармона, П. В. Парамонов, К. Ю. Федоровский, "О равномерной аппроксимации полианалитическими многочленами и задаче Дирихле для бианалитических функций”, Матем. сб., 193:10 (2002), 75-98; англ. пер.: J. J. Carmona, P. V. Paramonov, K. Yu. Fedorovskii, "On uniform approximation by polyanalytic polynomials and the Dirichlet problem for bianalytic functions", Sb. Math., 193:10 (2002), 1469-1492.

[6] У. Рудин, Функционалъный анализ, Мир, М., 1975; пер. с англ.: W. Rudin, Functional analysis, McGraw-Hill, New York-Düsseldorf-Johannesburg, 1973.

[7] Ch. Pommerenke, Univalent functions, Vandenhoeck \& Ruprecht, Göttingen, 1975.

[8] К. Ю. Федоровский, "О равномерных приближениях функций $n$-аналитическими полиномами на спрямляемых контурах в $\mathbb{C} "$, Матем. заметки, 59:4 (1996), 604-610; англ. пер.: K. Yu. Fedorovskii, "Uniform $n$-analytic polynomial approximations of functions on rectifiable contours in $\mathbb{C} "$, Math. Notes, 59:4 (1996), 435-439.

[9] W. Rudin, "Boundary values of continuous analytic functions", Proc. Amer. Math. Soc., 7:5 (1956), 808-811.

[10] L. Carleson, "Representations of continuous functions", Math. Z., 66:1 (1956), 447-451.

[11] П. Кусис, Введение в теорию пространств $H^{p}$ с приложением доказательства Волфба теоремы о короне, Мир, М., 1984; пер. с англ.: Р. Koosis, Introduction to $H^{p}$ spaces with an appendix on Wolff's proof of the corona theorem, London Math. Soc. Lecture Note Ser., 40, Cambridge Univ. Press, Cambridge-New York, 1980.

[12] Г. М. Голузин, Геометрическая теория функиий комплексного переменного, Наука, М., 1966; англ. пер.: G. M. Goluzin, Geometric theory of functions of a complex variable, Transl. Math. Monogr., 26, Amer. Math. Soc., Providence, RI, 1969.

[13] А.И. Маркушевич, Теория аналитических функиий, m. 2. Дальнейшее построение теории, 2-е изд., Наука, М., 1968.

[14] Дж. Гарнетт, Ограниченные аналитические функиии, Мир, М., 1984; пер. с англ.: J.B. Garnett, Bounded analytic functions, Pure Appl. Math., 96, Academic Press, New York-London, 1981.

\section{М. Я. Мазалов (М. Yа. Mazalov)}

Поступила в редакцию

Военная академия войсковой противовоздушной обороны 03.02.2009

Вооруженных Сил Российской Федерации им. Маршала

Советского Союза А. М. Василевского, г. Смоленск

E-mail: maksimmazalov@yandex.ru 